\title{
RIESZ SEMINORMS WITH FATOU PROPERTIES
}

\author{
C. D. ALIPRANTIS
}

ABSTRACT. A seminormed Riesz space $L_{\rho}$ satisfies the $\sigma-\mathrm{Fatou}$ property (resp. the Fatou property) if $\theta \leq u_{n} \uparrow u$ in $L$ (resp. $\theta \leq u_{\alpha} \uparrow u$ in $L$ ) implies $\rho\left(u_{n}\right) \uparrow \rho(u)$ (resp. $\rho\left(u_{a}\right) \uparrow \rho(u)$ ). The following results are proved:

(i) A normed Riesz space $L_{\rho}$ satisfies the $\sigma-F$ atou property if, and only if, its norm completion does and $L_{\rho}$ has $(A, 0)$.

(ii) The quotient space $L_{\rho} / I_{\rho}$ has the Fatou property if $L_{\rho}$ is Archimedean with the Fatou property. ( $\left.I_{\rho}=\{u \in L: \rho(u)=0\}_{\text {. }}\right)$

(iii) If $L_{\rho}$ is almost $\sigma$-Dedekind complete with the $\sigma-$ Fatou property, then $L_{\rho} / I_{\rho}$ has the $\sigma-$ Fatou property.

A counterexample shows that (iii) may be false for Archimedean Riesz spaces.

1. Riesz seminorms. For notation and terminology not explained below we refer the reader to [5]. A seminormed Riesz space $L_{\rho}$ is a Riesz space $L$ equipped with a seminorm $\rho$ satisfying $\rho(u) \leq \rho(v)$ whenever $|u| \leq|v|$ holds in $L$.

For seminormed Riesz spaces $L_{\rho}$ the following properties were introduced:

$(A, 0): u_{n} \downarrow \theta$ in $L$ and $\left\{u_{n}\right\}$ is a $\rho$-Cauchy sequence implies $\rho\left(u_{n}\right) \rightarrow 0$.

$(\mathrm{A}, \mathrm{i}): u_{n} \downarrow \theta$ in $L$ implies $\rho\left(u_{n}\right) \rightarrow 0$.

(A, ii): $u_{\alpha} \downarrow \theta$ in $L$ implies $\rho\left(u_{\alpha}\right) \rightarrow 0$.

Following Luxemburg and Zaanen [4, Notes II and XIII] we also have:

Definition 1.1 ( $\sigma$-Fatou property). A seminormed Riesz space $L_{\rho}$ satisfies the $\sigma$-Fatou property whenever $\theta \leq u_{n} \uparrow u$ in L implies $\rho\left(u_{n}\right) \uparrow \rho(u)$. (Fatou property). A seminormed Riesz space $L_{\rho}$ satisfies the Fatou property whenever $\theta \leq u_{a} \uparrow u$ in $L$ implies $\rho\left(u_{\alpha}\right) \uparrow \rho(u)$.

Obviously the Fatou implies the $\sigma$-Fatou, $(A, i)$ implies the $\sigma$-Fatou and $(A, i i)$ implies the Fatou property. Also the $\sigma$-Fatou implies the $(A, 0)$ property. Indeed, if $\left\{u_{n}\right\}$ is a $\rho$-Cauchy sequence w ith $u_{n} \downarrow \theta$ in $L$, then

Received by the editors September 14, 1973.

AMS (MOS) subject classifications (1970). Primary 46A40; Secondary 46B99.

Key words and phrases. Riesz spaces, $\sigma-\mathrm{F}$ atou property, norm completion, quotient Riesz spaces. 
$\theta \leq u_{m}-u_{n} \uparrow_{n \geq m} u_{m}$ in $L$, for each fixed $m$, and hence $\rho\left(u_{m}-u_{n}\right) \uparrow_{n \geq m}$ $\rho\left(u_{m}\right)$. This implies $\rho\left(u_{n}\right) \rightarrow 0$.

Example 1.2. (i) Let $L$ be the Riesz space of all real sequences which are eventually constant. Let $\rho(u)=|u(\infty)|+\sup \left\{\left|u_{n}\right|: n=1,2, \cdots\right\}$ for all $u \in L . \quad(u(\infty)=u(n)$ for all sufficiently large $n$.) Note that the $\sigma$-Fatou property does not hold in $L_{\rho}$. However $L_{\rho}$ does satisfy the $(A, 0)$ property.

(ii) Let $L$ be as in (i) and let $\rho(u)=\sup \{|u(n)|: n=1,2, \cdots\}$ for all $u$. Then $L_{\rho}$ is noncomplete with the Fatou property. Note that $(A, i)$ does not hold.

(iii) Let $L$ be the Riesz space of all bounded real valued Lebesgue measurable functions defined on $[0,1]$, with $f \leq g$ if $f(x) \leq g(x)$ for all $x \in$ $[0,1]$. Let $\rho(u)=\int_{0}^{1}|u(x)| d x+\sup \{|u(x)|: x \in[0,1]\}$ for all $u \in L$. Note that $L$ is $\rho$-complete with the $\sigma$-Fatou property but without the Fatou property.

(iv) The cartesian product of the spaces in (ii) and (iii) with the product norm gives a noncomplete normed Riesz space without the Fatou and $(A, i)$ properties, but with the $\sigma$-Fatou property. $\square$

We recall that a Riesz subspace $L$ of a Riesz space $M$ is said to be order dense in $M$ if $\sup \{v \in L: \theta \leq v \leq u\}=u$ holds in $M$ for all $u \in M^{+}$. If $M$ is Archimedean (and hence so is $L$ ) then the universal completion of $M$ [5, pp. 338-341] equally serves as the universal completion of $L$; consequently $M$ can be considered as a Riesz subspace of the universal completion of $L$. Now, if $L_{\rho}$ is a normed Riesz space with $(A, 0)$ then $L_{\rho}$ is order dense in its norm completion $\bar{L}_{\rho}\left[3\right.$, Theorem 61.5, p. 652] and so $\bar{L}_{\rho}$ "seats" in the universal completion of $L$ as an order dense Riesz subspace. This observation will be used in the next theorem.

Theorem 1.3. If the normed Riesz space $L_{\rho}$ satisfies the $\sigma$-Fatou property, then we have:

(i) The norm completion $\bar{L}_{\rho}$ of $L_{\rho}$, satisfies the $\sigma$-Fatou property.

(ii) $\rho(u)=\inf \left\{\lim \rho\left(u_{n}\right):\left\{u_{n}\right\} \subseteq L^{+}, u_{n} \uparrow\right.$ and $u_{n} \wedge|u| \uparrow|u|$ in $\left.\bar{L}_{\rho}\right\}$, for every $u$ in $\bar{L}_{\rho}$.

Proof. Let $K$ be the universal completion of $L$ [5, Theorem 50.8, p. 340]. Define $\lambda$ on $K$ by the formula:

$$
\lambda(u)=\inf \left\{\lim \rho\left(u_{n}\right):\left\{u_{n}\right\} \subseteq L^{+} ; u_{n} \uparrow \text { and } u_{n} \wedge|u| \uparrow|u| \text { in } K\right\}
$$

with inf $\varnothing=+\infty$. Then we have:

(i) $\lambda(u)=\rho(u)$ for all $u$ in $L$. 
To verify (i) use the $\sigma$-Fatou property of $\rho$.

(ii) $\lambda(u)=\lambda(|u|)$ for all $u$ in $K$, and $\theta \leq u \leq v$ in $K$ implies $\lambda(u) \leq \lambda(v)$.

(iii) $\lambda(u) \geq 0$ for all $u$ in $K$ and $\lambda(u)=0$ implies $u=\theta$.

To see (iii) use the order density of $L$ in $K$.

(iv) $\lambda(u+v) \leq \lambda(u)+\lambda(v), \lambda(\alpha u)=|\alpha| \lambda(u)$ for all $u, v$ in $K$ and all $\alpha$ in $\mathbf{R}$.

(v) If $\left\{u_{n}\right\} \subseteq L^{+}$and $\theta \leq u_{n} \uparrow u$ in $K$, then $\rho\left(u_{n}\right) \uparrow \lambda(u)$.

(vi) Let $U=\left\{u \in K^{+}: \theta \leq u_{n} \uparrow u\right.$, for some sequence $\left.\left\{u_{n}\right\} \subseteq L^{+}\right\}$. Assume $\theta \leq u_{n} \uparrow$ in $K,\left\{u_{n}\right\} \subseteq U$ and $\lambda\left(u_{n}\right) \uparrow \alpha<+\infty$. Then $\theta \leq u_{n} \uparrow u$ in $K$ and $\lambda(u)=\alpha$ for some $u$ in $U$.

To see (vi) pick $\left\{u_{n, k}: k=1,2, \cdots\right\} \subseteq L^{+}$such that $u_{n, k} \uparrow_{k} u_{n}(n=$ $1,2, \cdots)$. Define $w_{n}=\sup \left\{u_{i, n}: i=1, \cdots, n\right\} \in L^{+}(n=1,2, \cdots)$ and note that $\rho\left(w_{n}\right) \leq a$ for all $n$. Now, let $\theta<v \in L$. Pick $m \in N$ such that $m \rho(v)=$ $\rho(m v)>\alpha$, and observe that $w_{n} \wedge m v \uparrow m v$ implies $\rho(m v) \leq \alpha$. So, $\sup \left\{w_{n} \wedge m v: n=1,2, \cdots\right\}<m v$. This observation implies $\theta \leq w_{n} \uparrow u$ in $K\left[2\right.$, Proposition 1, p. 342]. (Since $E$ is order dense in $C_{\infty}(X)$, observe that Fremlin's proof works if we replace the assumption "for every $x>0$ in $C_{\infty}(X)$ " by "for every $x>0$ in $E$ ".) Thus $\theta \leq w_{n} \uparrow u$ and $u \in U$. Now, combine (v) with the relation $w_{n} \leq u_{n}$ for all $n$ to obtain $\theta \leq u_{n} \uparrow u$ and $\lambda(u)=\alpha$.

(vii) Let $\theta \leq u, \lambda(u)<+\infty$ and let $\epsilon>0$. Then there exists $v \in U$, $u \leq v$ such that $\lambda(v) \leq \lambda(u)+\epsilon$.

To verify (vii), pick $\left\{u_{n}\right\} \subseteq L^{+}, u_{n} \uparrow, u_{n} \wedge|u| \uparrow|u|$ and such that $\lim \rho\left(u_{n}\right) \leq \lambda(u)+\epsilon$. As in case (vi) note that $u_{n} \uparrow v$ in $K$ for some $v$ of $U$. Now use (v) to obtain $\lambda(v) \leq \lambda(u)+\epsilon$.

(viii) Let $L_{\lambda}=\{u \in K: \lambda(u)<+\infty\}$. Then $L_{\lambda}$ is a complete normed Riesz space.

For (viii) use (vii) and a routine argument to show that $L_{\lambda}$ satisfies the Riesz-Fischer property and hence it is $\lambda$-complete $[4$, Theorem 26.3, Note VIII, p. 105].

(ix) The closure of $L_{\rho}$ in $L_{\lambda}, \bar{L}_{\rho}$, is the norm completion of $L_{\rho}$.

Now, let $\theta \leq u_{n} \uparrow u$ in $\bar{L}_{\rho}$. Since $L$ is order dense in $K, u_{n} \uparrow u$ also holds in $K$. Given $\epsilon>0$, pick an element $u_{0}$ in $\bar{L}_{\rho}, u \leq u_{0}, u_{0} \in U$ with $\lambda\left(u_{0}-u\right)<\epsilon\left(\right.$ see [3, Theorem 60.3, p. 648]). Similarly pick $v_{n}$ in $\bar{L}_{\rho}, u_{n} \leq$ $v_{n} \leq u_{0}, \lambda\left(v_{n}-u_{n}\right) \leq \epsilon / 2^{n+1}$ and $v_{n} \in U, n=1,2, \cdots$. Put $w_{n}=\sup _{i} v_{i}$ : $i=1, \cdots, n\}(n=1,2, \cdots)$ and note $\lambda\left(w_{n}-u_{n}\right) \leq \epsilon$ and $u_{n} \leq w_{n} \leq u_{0}$ for all $n$. Hence $w_{n} \uparrow u_{1} \leq u_{0}$ in $L_{\lambda}$ and so $u \leq u_{1} \leq u_{0}$ in $L_{\lambda}$. But then $\lambda(u) \leq \lambda\left(u_{1}\right)=\lim \lambda\left(w_{n}\right) \leq \lim \lambda\left(u_{n}\right)+\epsilon$ for all $\epsilon>0$. Hence $\lambda\left(u_{n}\right) \uparrow \lambda(u)$, i.e., 
$\bar{L}_{\rho}$ satisfies the $\sigma$-Fatou property. Part (ii) follows immediately from the above construction.

Corollary 1.4. Let $L_{\rho}$ be a normed Riesz space with norm completion $\bar{L}_{\rho}$. Then the following statements are equivalent.

(i) $L_{\rho}$ satisfies the $\sigma$-Fatou property.

(ii) $L_{\rho}$ satisfies the $\sigma$-Fatou property and $L_{\rho}$ has $(A, 0)$.

Proof. To see that (ii) implies (i) use Theorem 61.5 of $[3$, p. 652].

For $L=C_{[0,1]}$ and $\rho(u)=\int_{0}^{1}|u(x)| d x$ we have $\bar{L}_{\rho}=L_{1}([0,1])$. Note that $\bar{L}_{\rho}$ satisfies the $\sigma$-Fatou property (in fact the $(A, i i)$ property). However, $L_{\rho}$ does not satisfy the $(A, 0)$ property [5, Exercise 18.14(i), p. 104].

We close this section recalling a notion useful for the next section. A Riesz space $L$ is called almost $\sigma$-Dedekind complete if it can be embedded as a super order dense Riesz subspace of a $\sigma$-Dedekind complete Riesz space $K$, i.e., if $L$ is a Riesz subspace of $K$ (more precisely $L$ is Riesz isomorphic to a Riesz subspace of $K$ ) such that for every $\theta \leq u \in K$, there exists a sequence $\left\{u_{n}\right\} \subseteq L$ with $\theta \leq u_{n} \uparrow u$ in $K$ (see [1]).

2. The quotient Riesz space. $L_{\rho} / I_{\rho^{*}}$. The null ideal of a given seminormed Riesz space $L_{\rho}$ is denoted by $I_{\rho}$, i.e., $I_{\rho}=\{u \in L: \rho(u)=0\}$. It is evident that $I_{\rho}$ is a $\sigma$-ideal (resp. a band) if $\rho$ satisfies the $\sigma$-Fatou property (resp. the Fatou property). It is also obvious that the quotient Riesz space $L_{\rho} / I_{\rho}$ becomes a normed Riesz space under the norm $[\rho]([u])=\rho(u)$. ( $[u]$ denotes the equivalence class of $u$.)

Question: If $L_{\rho}$ satisfies the $\sigma$-Fatou property, does the normed Riesz space $L_{\rho} / I_{\rho}$ satisfy the $\sigma$-Fatou property?

The next theorem gives a condition for the answer to be affirmative.

Theorem 2.1. Assume that the seminormed Riesz space $L_{\rho}$ satisfies the $\sigma$-Fatou property and that $L$ is almost $\sigma$-Dedekind complete. Then the normed Riesz space $L_{\rho} / I_{\rho}$ satisfies the $\sigma$-Fatou property.

Proof. Let $K$ be a $\sigma$-Dedekind complete Riesz space containing $L$ as a super order dense Riesz subspace. We can assume that the ideal generated by $L$ is all of $K$. Given $u \in K$ pick $\left\{u_{n}\right\} \subseteq L$ with $\theta \leq u_{n} \uparrow|u|$ in $K$ and define $\lambda(u)=\lim \rho\left(u_{n}\right)$. Note that $\lambda(u)$ is independent of the sequence chosen and that $\lambda$ is a Riesz seminorm of $K$ with the $\sigma$-Fatou property and with $\lambda=\rho$ on $L$.

Let $L / I_{\lambda}$ be the canonical image of $L$ in $K_{\lambda} / I_{\lambda}$. Observe that $L_{\rho} / I_{\rho}$ 
is Riesz isomorphic to $L / I_{\lambda}$ (the mapping $[u]=u+I_{\rho} \rightarrow u+I_{\lambda}=[u]$ does it) and that the quotient norm $[\rho]$ on $L_{\rho} / I_{\rho}$ and the norm induced from $K_{\lambda} / I_{\lambda}$ to $L / I_{\lambda}$ coincide. Now let $[\theta] \leq\left[u_{n}\right] \uparrow[u]$ in $L_{\rho} / I_{\rho}$, so $[\theta] \leq\left[u_{n}\right] \uparrow[u]$ holds also in $L / I_{\lambda}$. We can assume $\theta \leq u_{n} \uparrow \leq u$ in $L$, so $\theta \leq u_{n} \uparrow v \leq u$ holds in $K$ and hence $[\theta] \leq\left[u_{n}\right] \uparrow[v]$ in $K_{\lambda} / I_{\lambda}[5$, Theorem 18.11, p. 103]. Since $L / I_{\lambda}$ is order dense in $K_{\lambda} / I_{\lambda},\left[u_{n}\right] \uparrow[u]$ also holds in $K_{\lambda} / I_{\lambda}$ and hence $[\nu]=[u]$, so $\lambda(v)=\lambda(u)=\rho(u)$.

Thus $[\rho]\left(\left[u_{n}\right]\right)=\rho\left(u_{n}\right)=\lambda\left(u_{n}\right) \uparrow \lambda(v)=\rho(u)=[\rho]([u])$, and the proof is finished.

Question: If we replace the almost $\sigma$-Dedekind completeness of $L$ by Archimedeanness is Theorem 2.1 still true?

The following example shows that the answer is negative in general.

Example 2.2. Let $L$ be the Archimedean Riesz space $C\left(\mathbf{R}_{\infty}\right) .\left(\mathbf{R}_{\infty}\right.$ is the one point compactification of the real numbers considered with the discrete topology (see [5, Example (v), p. 141]). Note that $L$ is not almost $\sigma$-Dedekind complete. Now, define the Riesz seminorm $\rho$ on $L$, by $\rho(u)=|u(\infty)|+\sup \{|u(n)|: n=1,2, \cdots\}$. Note that $\rho$ satisfies the $\sigma$-Fatou property but not the Fatou property. (In fact $\rho$ satisfies the $(A, i)$ property.) Note also that $I_{\rho}$ is a band.

Now, let $u_{n}=\chi_{\{1, \cdots, n\}}, n=1,2, \cdots$. Then $\theta \leq u_{n} \uparrow \leq e$ in $L(e(x)=$ 1 for all $x \in \mathbf{R})$ and $\rho\left(u_{n}\right)^{n}=1$ for all $n$. It is easily seen that $[\theta] \leq\left[u_{n}\right] \uparrow[e]$ holds in $L_{\rho} / I$. But

$$
[\rho]\left(\left[u_{n}\right]\right)=\rho\left(u_{n}\right)=1 \nmid[\rho]([e])=\rho(e)=2 .
$$

Hence $L_{\rho} / I_{\rho}$ does not satisfy the $\sigma$-Fatou property.

A better situation holds if $\rho$ satisfies the Fatou property. The next theorem tells us that $L_{\rho} / I_{\rho}$ satisfies the Fatou property if $L_{\rho}$ does.

Theorem 2.3. Let $L_{\rho}$ be an Archimedean seminormed Riesz space with the Fatou property. Then the normed Riesz space $L_{\rho} / I_{\rho}$ satisfies the Fatou property.

Proof. Repeat the proof of Theorem 2.1 replacing $K$ by $L^{\delta}$, the Dedekind completion of $L . \square$

\section{REFERENCES}

1. C. D. Aliprantis and Eric Langford, Almost $\sigma-D e d e k i n d$ complete Riesz spaces and the main inclusion theorem, Proc. Amer. Math. Soc. 44(1974), 421-426.

2. D. H. Fremlin, On the completion of locally solid vector lattices, Pacific J. Math. 43 (1972), 341-347. 
3. W. A. J. Luxemburg, Notes on Banach function spaces. XVIa, Nederl. Akad. Wetensch. Proc. Ser. A 68 = Indag. Math. 27 (1965), 646-657. MR 32 \#6202e.

4. W. A. J. Luxemburg and A. C. Zaanen, Notes on Banach function spaces. II, VIII, XIII, Nederl. Akad. Wetensch. Proc. Ser. A $66=$ Indag. Math. 25 (1963), 148153; ibid. A 67 = Indag. Math. 26 (1964), 104-119, 530-543. MR $26 \# 6723$ b; 28 \#5324b; 30 \#3381b.

5.

. Riesz spaces. I, North-Holland, Amsterdam, 1971.

DEPARTMENT OF MATHEMATICS, CALIFORNIA INSTITUTE OF TECHNOLOGY, PASADENA, CALIFORNIA 91109

Current address: STD Research Corporation, 150 E. Foothill, Arcadia, California 91006 and Department of Mathematics, Occidental College, Los Angeles, California 90041 